\title{
A comparative study on the effects of fentanyl and dexmedetomidine on hemodynamic changes in patients undergoing brain tumor surgery in Al-Zahra Hospital in 2017
}

\author{
SEYED TAGHI HASHEMI ${ }^{1}$, MEHRDAD MASOUDIFAR ${ }^{2 *}$, ZOHREH RAHIMI ${ }^{3}$ \\ ${ }^{1}$ Assistant Professor, Anesthesiologist, and Intensive Care Unit Specialist, Medical School, Isfahan University of Medical Sciences, Isfahan, \\ Iran. \\ ${ }^{2}$ Associate Professor, Anesthesiologist, and Intensive Care Unit Specialist, Neuroanesthesia Fellowship, Medical School, Isfahan University \\ of Medical Sciences, Isfahan, Iran. \\ ${ }^{3}$ Professional Medical Doctor, Medical School, Isfahan University of Medical Sciences, Isfahan, Iran.
}

\begin{abstract}
Background: Considering the importance of maintaining hemodynamic stability in the patient undergoing brain tumor surgery, this study aimed to compare the effects of fentanyl and dexmedetomidine on hemodynamic changes in patients undergoing brain tumor surgery.

Methods: In a clinical trial study, 70 patients undergoing brain surgery were distributed in two groups of 35 . The first group received $5 \mathrm{mg} / \mathrm{kg}$ fentanyl, and the second group received $1 \mathrm{mg} / \mathrm{kg}$ dexmedetomidine immediately after induction of anesthesia. During the operation, the patients were evaluated for hemodynamic parameters, blood gas levels and coagulation profile during operation and recovery and compared between the two groups.

Findings: The mean changes of hemodynamic parameters was not differ between the two groups. Among blood gases HCO3had a higher level of in the dexmedotomidine group before, during and after surgery, and its changes during the study period were significant between the two groups $(P<0.001)$. Also, $P T$ level was higher in the dexmedmotidine group before surgery $(P=0.007)$, but its change was not significantly different during the study period $(P=0.14)$.

Discussion: Our findings showed that the use of dexmeditomidine with more favorable hemodynamic stability, shorter hemodynamic presentation, and shorter duration of stay of patients undergoing cerebral surgery. Considering the serious side effects of doxedetomidine administration, it seems that the use of Dexmedetomidine is preferred in patients undergoing brain surgery than fentanyl.
\end{abstract}

Keywords: Brain tumor, Dexmedmotidine, Fentanyl, Hemodynamic

\section{INTRODUCTION}

Complications after brain tumor surgery may have destructive impacts even after non-accidental operations. These complications are common, and in one study, $54.5 \%$ of patients who underwent extubation within 4 hours of surgery showed at least one complication $(1,2)$. The most common complications are postoperative nausea and vomiting, which occur in about $0.70 \%$ of craniotomy operations and cause a delay in hospital discharge, reducing patient satisfaction, and increasing the need for postoperative care. Headache is one of the most common complications, occurring in about $0.91 \%$ of patients with neurosurgery immediately after craniotomy due to surgical methods and meningeal stimulation $(3,4)$. A research reported new neurological problems and exacerbation of previous symptoms as the second most common problem after craniotomy (5). Delayed extubation is another complication, accounting for about $49.8 \%$ of craniotomy surgeries due to brain tumors. It is associated with increased rate of pneumonia, ICU cares, longer hospital stays, and higher hospital costs (6). Due to crucial events during craniotomy surgery such as fluid shift and excessive bleeding, the possibility of hemodynamic disorders during the operation is high, so the control of these parameters and prevention of increase or decrease in blood pressure and heart rate is vital.

Given the type of operation and the mentioned conditions, the use of a pre-medication that can minimize the occurrence of these disorders seems reasonable. However, high consumption of drugs used for this purpose and duration of the craniotomy surgery, which is usually high, can strongly affect brain function during and after surgery (10). The use of preoperative $\alpha 2$ receptor agonists improves hemodynamics and stability due to its several beneficial effects, including analgesic effects, inhibition of sympathetic outputs, anti-anxiety properties, and reduction of norepinephrine levels. It also has positive effects on myocardial oxygen supply and cardiac oxygen demand, thereby protecting the myocardium $(7,8)$. Dexmedetomidine is a drug with a high tendency to a2 receptor, so that the drug tendency to bind to $\alpha 2$ receptor versus $\alpha 1$ is at a ratio of 1600 to 1 (9). The use of dexmedetomidine before anesthesia has a positive impact on hemodynamic stability (10), associated with reduced postoperative mortality and reduced incidence of postoperative problems (11). The protective effects of dexmedetomidine on postoperative heart damages have been confirmed (12). In addition, decreased use of sedatives and analgesics has also been reported with dexmedetomidine (12). The aim of present study was to evaluate the effects of dexmedetomidine on hemodynamic parameters (systolic and diastolic blood pressures and heart rate) and the level of intraoperative bleeding in patients with dexmedetomidine with femoral shaft fractures, undergoing general anesthesia. Since there is no study available on the impact of dexmedetomidine infusion on hemodynamic parameters and level of intraoperative bleeding in patients undergoing craniotomy surgery, this study was conducted with the aim of comparing the effects 
of fentanyl and dexmedetomidine on hemodynamic changes in the patient underwent brain tumor surgery.

\section{MATERIALS AND METHODS}

The present study is a clinical trial conducted in 2017 and 2018 in Al-Zahra Educational Center. The statistical population of the study included the patients who were candidates for craniotomy surgery and were hospitalized in the center. Inclusion criteria of the study included age range of 18-65 years, $A S A=2,3$, not receiving drugs affecting hemodynamic status, not receiving anticoagulants, and patient's willingness to participate in the study. Also, unstable hemodynamic status at the beginning of the surgery and change in the surgical plan in terms of surgical technique were considered as exclusion criteria. Sample size was calculated to be 35 people in each group according to sample size formula to compare the means and considering 95\% confidence level, $80 \%$ test power, standard deviation of time of remaining in recovery that is about 1.17 minutes and the minimum significant difference between the two groups that was considered 0.8 . All patients received the same amount of fluid the night before the surgery. They were in NPO for 8 hours before the operation and received 1 liter, $1 / 3$, and $2 / 3$ of the serum every 8 hours. Pre-medication of patients in both groups was similar and it was $10 \mathrm{mg}$ diazepam tablet, taken the night before surgery. During the operation, elastic bandages were used on both legs in both groups.

Patients' characteristics were recorded in the prepared questionnaires. In this questionnaire, the patient's demographic characteristics (name and surname, age, gender, height and weight) as well as patient's hemodynamic indicators (blood pressure, heart rate and blood oxygen saturation and respiration rate) were recorded. The surgeon and the person responsible for completing the questionnaire were unaware of the group of the patient.

Hemodynamic characteristics of the patients (systolic blood pressure, diastolic blood pressure, heart rate and blood oxygen saturation) and respiratory rate before fluid therapy and during anesthesia were recorded in the questionnaire. Blood pressure in all patients was measured by a single person using a blood-pressure monitor. Blood oxygen saturation and heart rate were also measured with a pulse oximeter. After induction of anesthesia, they were anesthetized by the same method (fentanyl, cisatracurium, morphine, propofol, isoflurane and 02 / no2). Patients were randomly assigned to two groups using block randomized method and accordingly, 70 eligible patients were distributed in two groups (35 patients in each group). The first group received $5 \mathrm{mg} / \mathrm{kg}$ of fentanyl and the second group received $1 \mu \mathrm{g} / \mathrm{kg}$ of dexmedetomidine immediately after induction of anesthesia. In case of hypotension, first $5 \mathrm{mg} / \mathrm{kg}$ of each solution was given in the desired group and if there was no response, ephedrine would be administrated and the amount of fluid and ephedrine was recorded. During the operation, hemodynamic characteristics, including systolic blood pressure, diastolic blood pressure, mean arterial blood pressure, heart rate and central venous pressure were measured and recorded every 15 minutes. During monitoring, if systolic blood pressure drops to less than 90 $\mathrm{mm} \mathrm{Hg}, 5 \mathrm{mg}$ of ephedrine was administrated at 2-minute intervals and the number of administrations was recorded in the questionnaire. If the heart rate dropped to less than 45 beats per minute, $0.5 \mathrm{mg}$ of atropine was administrated into patient and recorded in the questionnaire. If the number of beats did not increase, the dose would be repeated at 2-minute intervals and the number of administrations was recorded in the questionnaire. $\mathrm{PH}$, $\mathrm{HCO} 3, \mathrm{PCO} 2$ levels of all patients were measured every 30 minutes during surgery and recovery and serum sodium, potassium and calcium levels were measured every two hours during surgery and recovery and coagulation tests including PT, PTT, INR were used before and after surgery.

The level of bleeding during the operation was calculated from the total blood suction and the number of gauze pads saturated with blood. Each gauze pad completely saturated with blood was considered equivalent to $20 \mathrm{cc}$ of bleeding. If the patient did not control hemodynamic or hemostatic disorders, his or her problem would record in the medical record and the necessary measures would be taken outside the mentioned protocols to rehabilitate the patient. Central venous pressure and coagulation profile including PT, PTT, and INR were also determined and recorded before, during and after surgery. After collecting the research data, they were entered into the computer and analyzed in SPSS24 software. To analyze the data, Chi-square statistical tests (to compare qualitative and nominal data between the two groups), $T$ test (to compare quantitative data between the two groups) and repeated measures ANOVA (to examine the trend of changes in variables over time in two groups) were used.

\section{RESULTS}

In the present study, 70 patients who underwent brain surgery in two groups (35 patients in each group) received fentanyl and dexmedetomidine were studied. No patient was excluded during the study due to unwanted complications. There was no significant difference between the two groups in terms of age and gender distribution, height, body mass index and ASA (Table 1).

Table 1: Distribution of demographic and clinical variables in patients of two groups

\begin{tabular}{|l|l|l|l|l|}
\hline Variable & \multicolumn{2}{|l|}{ Group } & P \\
\cline { 2 - 4 } & Fentanyl & Dexmedetomidine & \\
\hline Mean age (yea) & $4.13 \pm 1.52$ & $7.12 \pm 2.55$ & 33.0 \\
\hline Gender & Male & $21(60)$ & $4) .18(51$ & 47.0 \\
& female & $14(40)$ & $6) .17(48$ & \\
\hline Mean BMI & & $39.2 \pm 03.25$ & $87.2 \pm 86.25$ & 19 \\
\hline ASA & I & $7) .8(25$ & $4) .11(31$ & 77.0 \\
\cline { 2 - 4 } & II & $9) .22(62$ & $3) .19(54$ & \\
\hline III & $4) .4(11$ & $3) .5(14$ & \\
\hline
\end{tabular}

In the two groups receiving fentanyl and dexmedetomidine, the mean duration of operation was $145.6 \pm 32.1$ and $144.9 \pm 20.5$, respectively $(P=0.91)$. The mean duration of anesthesia in fentanyl and dexmedetomidine groups was $193.3 \pm 49.5$ and $179.7 \pm$ 27.5 , respectively $(P=0.16)$. The mean extubation time in fentanyl and dexmedetomidine groups was $29.6 \pm 11.5$ and $31.7 \pm 12.5$ minutes, respectively $(P=0.46)$. No significant difference was found between the groups in this regard (Diagram 1). 


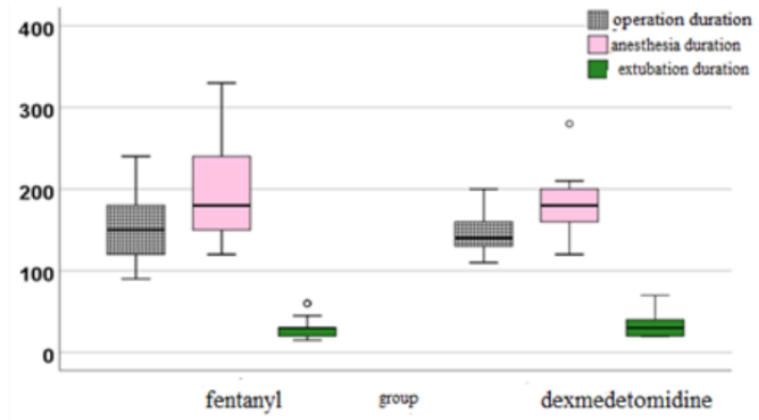

Diagram 1: Median, range, and $25-25 \%$ percentile of operation, anesthesia and extubation durations in two groups

Diagrams 2-6 show the trend of changes in patients' hemodynamic parameters before drug injection up to 30 minutes of recovery. According to repeated measures ANOVA, there was no significant difference between the two groups receiving fentanyl and dexmedetomidine.

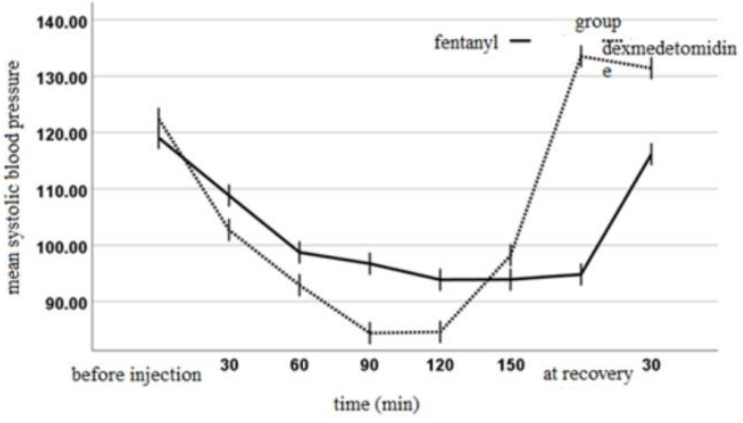

Diagram 2: Changes in systolic blood pressure from preoperative to recovery in two groups $(P=0.16)$

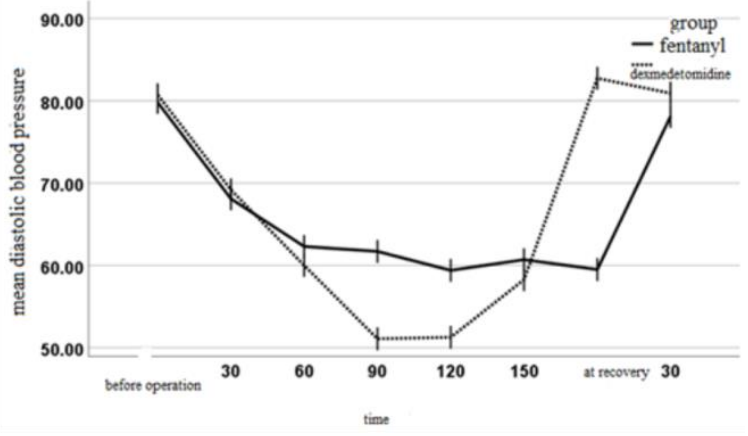

Diagram 3: Changes in diastolic blood pressure from preoperative to recovery in two groups $(P=0.86)$

Table 2 shows the mean and standard deviation of blood gas levels and coagulation profiles in the two groups. According to the results obtained, among the blood gases, the level of $\mathrm{HCO} 3$ in the dexmedetomidine group was significantly higher before, during and after the operation and the trend of its changes during the study period was significant between the two groups $(P<0.001)$. In addition, the level of PT before surgery was higher in the dexmedetomidine group $(P=0.007)$, but the trend of its changes during the study period did not differ significantly $(P=0.14)$

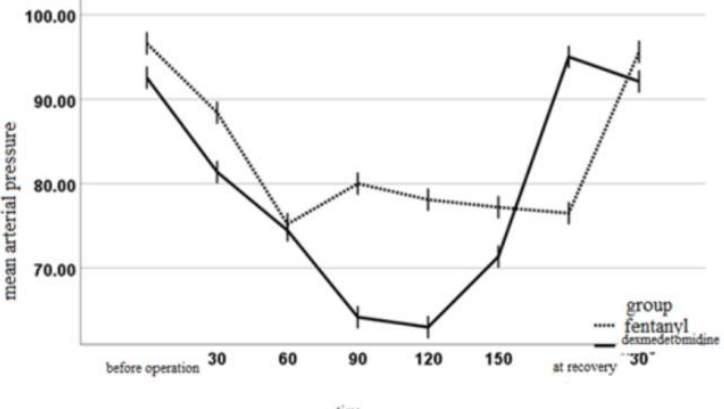

Diagram 4: Trend of changes in mean arterial pressure from preoperative to recovery in two groups $(P=0.22)$

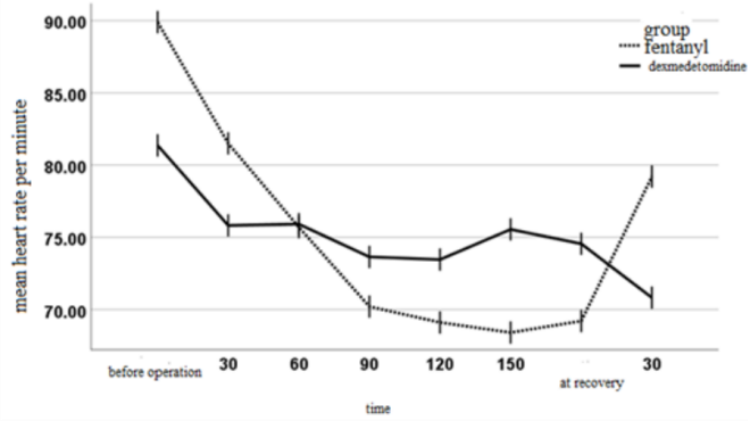

Diagram 5: Trend of heart rate changes from preoperative to recovery in two groups $(P=0.22)$

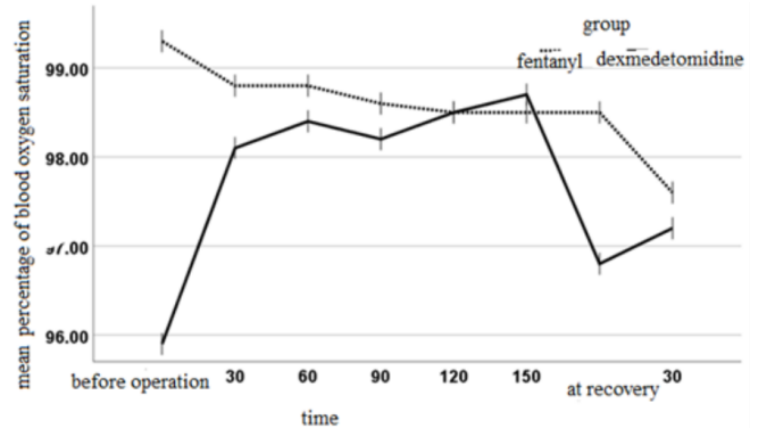

Diagram 6: Trend of changes in the percentage of blood oxygen saturation from preoperative to recovery in two groups $(P=0.09)$

Out of 70 patients, 39 (55.7\%) showed postoperative complications, 25 of which were in the fentanyl group and 15 were in the dexmedetomidine group (68.6\% vs. $42.9 \%$ ) and the incidence of complications was significantly lower in the dexmedetomidine group $(P=0.03)$. The type of complications was significantly different between the two groups $(P=0.001)$ (Diagram 7$)$. It should be noted that 1 patient in fentanyl group suffered hypotension and tachycardia simultaneously and 3 patients in the dexmedetomidine group suffered hypotension and bradycardia.

The mean length of staying in recovery in the two groups of fentanyl and dexmedetomidine was $124.6 \pm 26.4$ and $79.1 \pm 15.2$ minutes, respectively, and this time was significantly shorter in the dexmedetomidine group ( $P$ $<0.001)$. The mean volume of bleeding during operation in the two groups was $957.136 \pm 367.7$ and $157 \pm 118.5 \mathrm{ml}$, respectively, but no significant difference was observed 
between the two groups $(P=0.13)$. During the operation, 11 patients (15.7\%) received vasopressor, of which 5 (14.3\%) were in the fentanyl group and 6 (17.1\%) were in the dexmedetomidine group $(P=0.99)$. The type of received vasopressor included 7 cases of ephedrine (3 in the fentanyl group and 4 in the dexmedetomidine group) and 4 cases of phenylephrine ( 2 in each group). The doses of ephedrine and phenylephrine in all recipients were 5 and $50 \mathrm{mg}$, respectively.

Table 2: Mean and standard deviation of blood gas levels and coagulation profile in two groups

\begin{tabular}{|c|c|c|c|c|c|}
\hline Parameter & Time & Fentanyl Group & Dexmedetomidine group & P1 & P2 \\
\hline \multirow{3}{*}{$\mathrm{Hp}$} & Before operation & $7.38 \pm 0.05$ & $7.36 \pm 0.06$ & 0.298 & \multirow{3}{*}{0.11} \\
\hline & During operation & $7.4 \pm 0.07$ & $7.37 \pm 0.06$ & 0.069 & \\
\hline & End of recovery & $7.39 \pm 0.04$ & $7.38 \pm 0.05$ & 0.52 & \\
\hline \multirow{3}{*}{$\mathrm{HCO} 3$} & Before operation & $21.38 \pm 1.7$ & $20.71 \pm 3.5$ & 0.14 & \multirow{3}{*}{0.11} \\
\hline & During operation & $22.41 \pm 1.68$ & $21.76 \pm 3.25$ & 0.09 & \\
\hline & End of recovery & $21.29 \pm 1.87$ & $22.99 \pm 4.09$ & 0.32 & \\
\hline \multirow{3}{*}{ PCO2 } & Before operation & $36.96 \pm 2.82$ & $38.34 \pm 4.17$ & 0.15 & \multirow{3}{*}{0.25} \\
\hline & During operation & $38.12 \pm 5.63$ & $38.34 \pm 4.71$ & 0.87 & \\
\hline & End of recovery & $37.04 \pm 3.91$ & $38.45 \pm 4.37$ & 0.19 & \\
\hline \multirow{3}{*}{ PT } & Before operation & $10.56 \pm 0.7$ & $11.09 \pm 0.77$ & 0.007 & \multirow{3}{*}{0.14} \\
\hline & During operation & $11.07 \pm 0.93$ & $11.02 \pm 0.76$ & 0.85 & \\
\hline & End of recovery & $11.12 \pm 0.91$ & $11.12 \pm 0.75$ & 0.98 & \\
\hline \multirow{3}{*}{ PTT } & Before operation & $33.9 \pm 4.4$ & $32.1 \pm 4.5$ & 0.12 & \multirow{3}{*}{0.65} \\
\hline & During operation & $32.4 \pm 4.9$ & $32.4 \pm 4.6$ & 0.97 & \\
\hline & End of recovery & $33.1 \pm 4.9$ & $32.8 \pm 4.4$ & 0.84 & \\
\hline \multirow{3}{*}{ INR } & Before operation & $1.16 \pm 0.08$ & $1.13 \pm 0.06$ & 0.11 & \multirow{3}{*}{0.58} \\
\hline & During operation & $1.11 \pm 0.07$ & $1.13 \pm 0.06$ & 0.28 & \\
\hline & End of recovery & $1.1 \pm 0.05$ & $1.12 \pm 0.06$ & 17 & \\
\hline
\end{tabular}

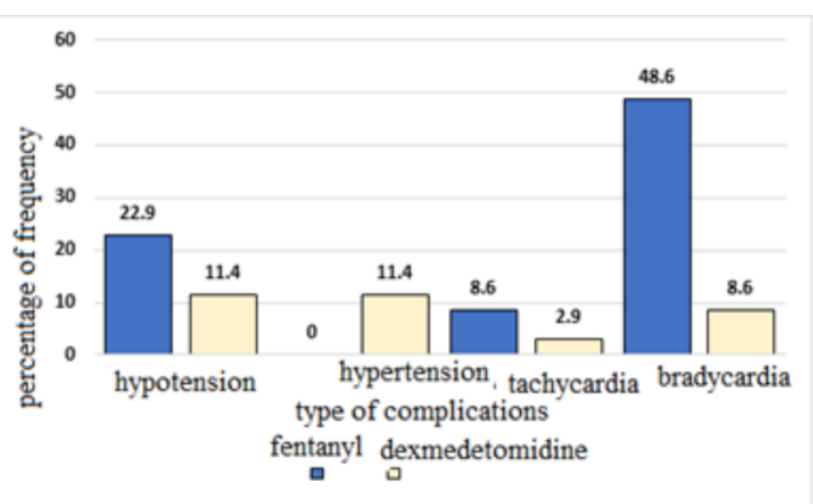

Diagram 7: Percentage of incidence of complications during operation in both groups

\section{DISCUSSION AND CONCLUSION}

Hemodynamic disorder is the most serious problem in patients undergoing surgery under general anesthesia. Anesthesiologists are responsible to take care of the patient's hemodynamic status and prevent these disorders. Although various methods have been proposed so far to maintain hemodynamic stability during surgery, its incidence varies in different conditions due to different conditions of patients, type of surgery, duration of surgery and depth of anesthesia. Moreover, no single and acceptable solution has been presented to prevent hemodynamic disorders. Dexmedetomidine is one of the anesthetic drugs, introduced to Iranian pharmaceutical market in recent years and research has shown that this drug is well tolerated by patients and can be effective in preventing postoperative complications and postoperative pain control in various surgeries, in addition to maintaining hemodynamic stability.
Since anesthesiologists commonly use fentanyl and dexmedetomidine currently and no comprehensive study has examined the effects of these two drugs on hemodynamics and postoperative complications of brain surgery, the present study was conducted with the aim of comparing the effects of fentanyl and dexmedetomidine on hemodynamic changes in patients undergoing brain tumor surgery at Al-Zahra Hospital in Isfahan in 2017 .According to the results of our study, the two groups did not differ significantly in terms of distribution of demographic and clinical variables, including age and gender distribution, ASA and body mass index, and the above-mentioned factors did not have a confounding effect on hemodynamic parameters, blood gas levels and coagulation profiles. Thus, the observed differences between the two groups were related to the type of drug used.

Hemodynamic parameters of patients, which were measured before operation, during operation, and 30 minutes after recovery did not show a significant difference between the two groups. In general, the trend of changes in hemodynamic parameters was similar in two groups, but the incidence of hemodynamic disorders in the dexmedetomidine group was lower than fentanyl group, and accordingly, dexmedetomidine use was associated with better hemodynamic stability during surgery and recovery. In a study conducted by Attari et al. (2018) in AlZahra Hospital in Isfahan, the effect of adding dexmedetomidine or fentanyl to marcaine in lumbar discectomy surgery was investigated. In the mentioned study, the marcaine-dexmedetomidine group showed more hemodynamic stability than the fentanyl-marcaine group and the incidence of hemodynamic complications was significantly lower in the marcaine-dexmedetomidine (13). In another research carried out by Pournajafian et al. ( 2017), the effect of dexmedetomidine plus remifentanil on hemodynamic changes during laparoscopic surgery was 
evaluated. In the mentioned study, the incidence of hemodynamic disorders was lower in dexmedetomidine group and patients in this group had less postoperative pain severity (14). In a research carried out by Naqibi et al, the effect of midazolam and dexmedetomidine on the prevention of cognitive impairment after cataract surgery, hemodynamic parameters did not differ significantly between the two groups and the incidence of hemodynamic disorders during surgery was lower in the dexmedetomidine group (15).

Investigating of coagulation factors and arterial blood gases before, during and after surgery did not show a significant difference between the two groups, so it could be stated that none of the above-mentioned drugs had a negative effect on coagulation factors in patients. In a research conducted by Mirzik et al, the use of dexmedetomidine in tonsillectomy surgery did not have an unfavorable effect on PT and PTT levels (16). Based on the results of our study, the length of staying in recovery was significantly shorter in dexmedetomidine group patients than that in the fentanyl group patients. Although the dexmedetomidine group had less bleeding volume, the difference between the two groups was not significant. In a research conducted by Attari et al, patients receiving marcaine-dexmedetomidine had a shorter recovery period than patients receiving fentanyl-marcaine, so it seems that the use of dexmedetomidine is associated with a faster return of patient to normal status. In a research conducted by Klamt et al, the effect of dexmedetomidine and fentanyl on heart surgery as examined and it was found that the use of dexmedetomidine was associated with lower hemodynamic stability and shorter length of stay in ICU (17).

\section{CONCLUSION}

Results of our research revealed that the use of dexmedetomidine is associated with better hemodynamic stability, less hemodynamic disorders and shorter hospital stay of patients undergoing brain surgery. Dexmedetomidine is preferable to fentanyl in patients undergoing brain surgery. However, due to the limitations of this study, such as small sample size, it is suggested to conduct more studies in this area.

\section{REFERENCES}

1. Bruder NJ. Awakening management after neurosurgery for intracranial tumours. Curr Opin Anaesthesiol 2002; 15(5): 477-82.

2. de Oliveira Ribeiro Mdo C, Pereira CU, Sallum AM, MartinsFilho PR, Desantana JM, da Silva Nunes M, et al. Immediate post-craniotomy headache. Cephalalgia 2013; 33(11): 897905.

3. Ferreira Kdos S, Dach F, Speciali JG. Scar neuromas as triggers for headache after craniotomy: clinical evidence. Arq Neuropsiquiatr 2012; 70(3): 206-9.

4. Wong JM, Panchmatia JR, Ziewacz JE, Bader AM, Dunn IF, Laws ER, et al. Patterns in neurosurgical adverse events: intracranial neoplasm surgery. Neurosurg Focus 2012; 33(5): E16. 6. Cai YH, Zeng HY, Shi ZH, Shen J, Lei YN, Chen BY, et al. Factors influencing delayed extubation after infratentorial craniotomy for tumour resection: a prospective cohort study of 800 patients in a Chinese neurosurgical centre. J Int Med Res 2013; 41(1): 208-17.

5. Pierre S, Benais H, Pouymayou J. Apfel's simplified score may favourably predict the risk of postoperative nausea and vomiting. Can J Anaesth 2002; 49(3): 237-42.

6. Haynes AB, Weiser TG, Berry WR, Lipsitz SR, Breizat AH, Dellinger EP et al. A surgical safety check list to reduce morbidity and mortality in a global population. $\mathrm{N}$ Engl $\mathrm{J}$ Med 2009; 360:491-499.

7. Fuhai Ji, Zhongmin Li, Hung Nguyen, Nilas Young, Pengcai Shi, Neal Fleming, Hong Liu. Perioperative Dexmedetomidine Improves Outcomes of Cardiac Surgery. Circulation.2013; 127: 1576-1584.

8. Jianjun Ren, Hijjun Zhang, Lining Huang, Yue Liu, Fengqin Liu and Zhenming Dong. Protective effect of dexmedetomidine in coronary artery bypass grafting surgery. Exp Ther Med.2013; 6: 497-502.

9. Bakhamees HS, El-Halafawy YM, El-Kerdawy HM, Gouda $\mathrm{NM}$, Altemyatt S. Effects of dexmedetomidine in morbidly obese patients undergoing laparoscopic gastric bypass. Middle East J Anesthesiol. 2007; 19:537- 51.

10. McVey JD, Tobias JD. Dexmedetomidine and ketamine for sedation during spinal anesthesia in children. $\mathrm{J}$ Clin Anesth 2010; 22(7): 538-45.

11. Selim MF, Elnabtity AM, Hasan AM. Comparative evaluation of epidural bupivacaine - dexmedetomidine and bupivacaine -fentanyl on Doppler velocimetry of uterine and umbilical arteries during labor. J Prenat Med 2012; 6(3): 47-54.

12. Al-Mustafa MM, Abu-Halaweh SA, Aloweidi AS, Murshidi MM, Ammari BA, Awwad ZM, et al. Effect of dexmedetomidine added to spinal bupivacaine for urological procedures. Saudi Med J 2009; 30(3): 365-70.

13. Attari M, Reisi-Dehkordi S, Ahanjideh F. Comparison of the Effect of Adding Dexmedmodine and Fentanyl to Bupivacaine on the Adequacy of Anesthesia and Postoperative Pain in Lumbar Discectomy under Spinal Anesthesia. J Isfahan Med Sch 2018; 36(473): 292-8.

14. Pournajafian A, Rokhtabnak F, Ghodraty M, Sadeghi F, Ghamari AA. Better Control of Hemodynamic Changes during Laparoscopic Cholecystectomy with Remifentanil Compared to Dexmedetomidine. J Isfahan Med Sch 2015; 33(356): 1810-9

15. Naghibi K, Shetabi H, Nasrollahi K, Mansouri N. The Effect of Intravenous Midazolam and Dexmedetomidine on Prevention of Cognitive Dysfunction after Cataract Surgery in the Elderly Patients under General Anesthesia Compared with the Control Group. J Isfahan Med Sch 2019; 36(504): 1395-400.

16. Mizrak A, Karatas E, Saruhan R, Kara F, Oner U, Saricicek $\mathrm{V}$, Baysal $\mathrm{E}$. Does dexmedetomidine affect intraoperative blood loss and clotting tests in pediatric adenotonsillectomy patients?. Journal of Surgical Research. 2013 Jan 1;179(1):94-8.

17. Klamt JG, Vicente WV, Garcia LV, Ferreira CA. Effects of dexmedetomidine-fentanyl infusion on blood pressure and heart rate during cardiac surgery in children. Anesthesiology research and practice. 2010;2010. 